\title{
The Magnetic white dwarfs in AM Her variables
}

\author{
D.T. Wickramasinghe \\ Department of Mathematics \\ Australian National University \\ CANBERRA A.C.T.
}

\section{\$1. Introduction}

There are now some 15 cataclysmic variables that have been recognised as being of the AM Her type. They are characterised by the presence of variable circular and linear polarisation in the optical and IR spectral regions, approaching $40 \%$ in some systems. The polarized radiation has been attributed to high harmonic cyclotron emission from hot $(\sim 5-30 \mathrm{Kev})$ plasma located at accretion shocks near the poles of a highly magnetised $(\sim 20-60 \mathrm{MG})$ white dwarf primary. There is hope that the study of the rich variety of phenomena exhibited by these systems will eventually enable us to probe the magnetic field structure of the underlying white dwarfs, perhaps even in more detail than has been possible with the isolated magnetic white dwarfs (Wickramasinghe (1987), Schmidt (1987)).

In this paper we review the theory of $A M$ Her variables concentrating on recent developments concerning the interpretation of intensity and polarisation observations. More general reviews covering other aspects can be found in a series of articles that have recently been published in the proceedings of the Vatican Observatory conference on circumstellar polarisation.

\section{\$2. The basic model and system characteristics}

The basic model that has been developed to explain these systems is shown schematically in Figure 1. The systems have the following characteristics :

- material leaving the inner Lagrangian point $L_{I}$ is chanelled via field lines directly onto the surface of the magnetic white dwarf without the formation of an accretion disc.

- the material impacts onto two extended regions EA and EB on the white dwarf surface where stand off shocks are formed.

- the shocks have temperature and density structure and have a variable height above the stellar surface which depends, in a complicated manner, on the specific accretion rate (Stockman (1988)). The hard X-rays are produced in a high density core covering a fractional area $f-10^{-5}$ of the white dwarf surface. (Buermann (1988)). The polarized optical-IR emission arises from a more extended (f $\sim 10^{-3}$ $10^{-4}$ ) and lower density region surrounding the $X$-ray core. (Bailey (1988), Schmidt (1988)). 
- The cyclotron and X-ray regions are surrounded by a low density accretion halo. The material in this region impacts directly on the white dwarf surface, evidently without the formation of a shock. The halo gives rise to non photospheric Zeeman split Balmer lines which are seen in absorption against the background cyclotron flux. (Wickramasinghe, Visvanathan and Tuohy (1987), Schmidt (1988)).

- Resolvable cyclotron emission features are sometimes seen from the accretion shocks. The spacing of the cyclotron harmonics gives a direct and accurate determination of the magnetic field strength at the shock. (Wickramasinghe and Meggitt (1982), Ferrario et al (1988), Ferrario and Wickramasinghe (1988)).

- the systems are observed in both high and low states. During a low state photospheric Zeeman lines are detected from the underlying magnetic white dwarf and phase dependent observations can be used to study the field structure. (Wickramasinghe and Martin (1985)).

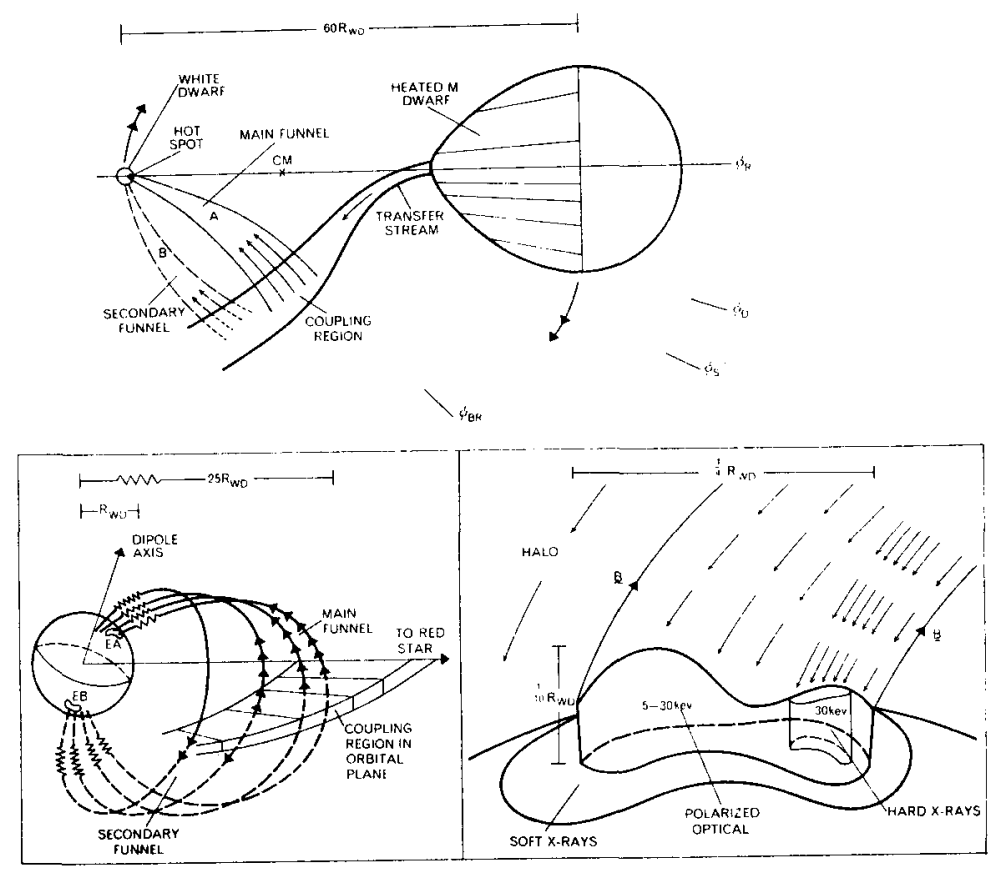

Figure 1 Top panel : View from above the orbital plane. Bottom left panel : The two accretion funnels leading to extended shocks EA and EB on the white dwarft surface. Bottom right panel : Structure of a shock. 
We proceed to discuss some of these characteristics in more detail concentrating in particular on the recent evidence for two pole emission and for extended shock structures.

\section{\$2.1 Accretion along closed field lines}

There is now overwhelming evidence in support of the view that there are two well separated cyclotron emission regions on the white dwarf surface. In systems such as AM Herculis and WV Puppis, the two regions are simultaneously visible for a part of the rotational cycle indicating that they are displaced by a significant amount from the magnetic poles (Wickramasinghe (1988), Meggitt and Wickramasinghe (1988), §2.3). The presence of such regions can be explained in one of three ways.

(i) If the coupling region has a small radial and azimuthal extent in the orbital plane, accretion may occur onto the foot points of a closed field line that lies entirely within the Roche equipotential. Otherwise accretion can only occur onto the closer foot point (Ferrario, Wickramasinghe and Tuohy (1988)). The situation is ilustrated in Figure 2.

(ii) If the coupling region has a small radial but large $\left(\sim 90^{\circ}\right)$ azimuthal extent in the orbital plane (as depicted in Figure 1), accretion may occur onto a second region from material that is coupled to field lines further downstream, even if the coupling radius is large and accretion cannot occur onto two spots along closed field lines. The two spots are expected to be centered on different magnetic longitudes and to exhibit linear extension mainly in the magnetic longitudinal direction.

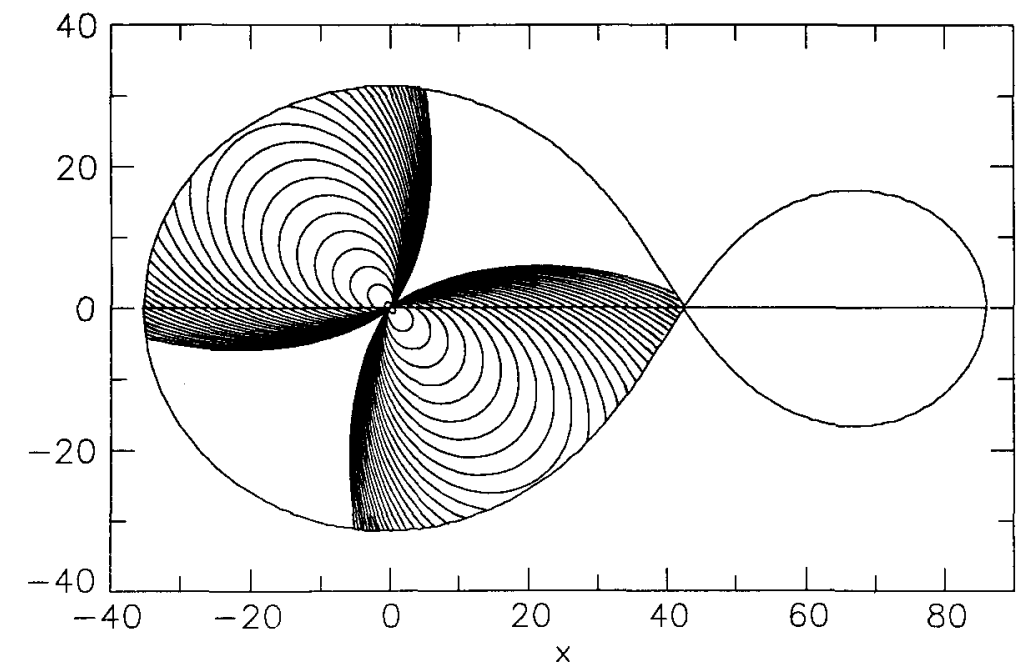

Figure 2 Closed field lines contained within the critical Roche lobe for a mass ratio $q=0.25$. The $Z$ axis is perpendicular to the orbital plane. 
(iii) If the coupling region has a large radial and small azimuthal extent, perhaps as a result of a clumpy accretion flow (Buermann (1988)), accretion may occur onto two spots which are on the same magnetic longitude. The spots are then expected to exhibit significant linear extension in the magnetic latitudinal direction and not be centered near the foot points of a single closed field line (Figure 2).

Clearly for a given dipole inclination $\theta_{d}$, a larger orbital period $P_{\text {orb }}$, or a smaller polar field strength $B_{p}$ will favour two pole accretion. Likewise, for a given $P_{\text {orb }}$ and $B_{p}$, two pole accretion is more likely in systems with smaller $\theta_{d}$.

The accretion pattern on the surface will also depend on $\theta_{d}$. The curves of constant magnetic pressure in the orbital plane will be nearly circular and will map onto the surface in a ribbon that follows curves of constant magnetic latitude. For an inclined dipole the curves of constant magnetic pressure will be oval and will map onto the surface in ribbons that cross curves of constant magnetic latitude. These would be the patterns expected in case (ii). However the actual shapes will depend on whether cases (i), (ii) or (iii) are more appropriate.

Meggitt and Wickramasinghe (1988) recently analysed the linear polarisation pulse and angle observations of VV Puppis and AM Herculis for spot positions assuming that they are located at the foot points of closed field lines. The resulting geometrical model gave results in good agreement with the circular polarisation observations and eclipse constraints supporting the idea of accretion along closed field lines. The small discrepancies between the model and observations could be resolved by assuming that the emission regions were slightly displaced in magnetic longitude as is expected for a coupling region of finite azimuthal extent in the orbital plane or if the dipole is offset from the centre as discussed by Wickramasinghe and Martin (1985). The model fits indicate that the shocks are displaced by $\sim 10^{\circ}$ from the magnetic poles (\$2.3).

A different picture emerged from an attempt to carry out a similar analysis of the linear polarisation observations of EF Eri. Meggitt and Wickramasinghe (1988) noted that the pulses and polarisation angles, if interpreted straightforwardly, suggested the presence of four spots on the white dwarf surface. They argued in favour of a geometrical model in which two of the spots were located near the magnetic poles and the other two roughly $90^{\circ}$ degrees away near the magnetic equator. Since the regions could not be connected by closed field lines in a dipole field distribution, they discussed the possibility of a predominantly quadrupolar field distribution. However, it has recently become apparent that it may also be possible to interpret these observations by assuming two extended ribbon like emission regions in an underlying dipolar field structure. We show in $\$ 2.2$ that a single ribbon, could under certain circumstances, mimic two spots in its polarisation properties. For this interpretation to be viable for EF 
Eri it is necessary for the emission regions to be extended mainly in magnetic latitude as is expected if the coupling region has a significant radial extension as in (iii) above or for the dipole to be strongly offset. We conclude that while the general idea of accretion along closed field lines appears to be correct for systems such as VV Puppis and AM Herculis, there are other systems where the situation may be more complicated.

\section{\$2.2 Properties of accretion ribbons}

The theory of cyclotron emission has been recently reviewed by Wickramasinghe (1988). We note that the total opacity $\tau_{\mathrm{k}}$ due to cyclotron absorption, in the collisionless approximation, is given by $\tau_{k}=\Lambda_{s} \kappa_{f}\left(T_{e}, \theta, \omega / \omega_{c}\right)$ where

$$
\Lambda_{\mathrm{s}}=2.0110^{6}\left(\mathrm{~s} / 10^{6} \mathrm{~cm}\right)\left(\mathrm{N}_{\mathrm{e}} / 10^{16} \mathrm{~cm}^{-3}\right)\left(\mathrm{B} / 3.10^{7} \mathrm{G}\right)^{-1}
$$

$\mathrm{N}_{e}$ and $\mathrm{T}_{e}$ are the electron number density and temperature respectively, $B$ is the magnetic field strength, $\omega_{c}=e B / m_{e} c$ is the cyclotron frequency, $s$ is the path length and $\theta$ is the angle between the direction of propagation and $\mathbf{B} . \kappa_{\mathbf{f}}$ is a strong function of $\mathrm{T}_{\mathrm{e}}, \theta$ and harmonic number $\mathrm{n}\left(=\omega / \omega_{\mathrm{c}}\right)$ and has a value $\sim 1-10$ near the cyclotron fundamental. Since $\Lambda_{\mathrm{s}}-10^{5}-10^{8}$ in the accretion shocks it follows that cyclotron opacity plays a dominant role in determining their radiation properties.

Early attempts at analysing observations were based on the assumption that the shocks were structureless and could be characterised by a point source with the same value of $\Lambda_{\mathrm{s}}$ at all angles $\theta$ to the field. The properties of these models can be summarised as follows :

- The slope of the energy distribution defined by the harmonic peaks is a strong function of frequency in the optically thin (high harmonic) regime. At $\theta=90^{\circ}$, $\mathrm{I}_{\omega} \propto \omega^{\mathrm{p}}$ where $\mathrm{p}=-8,-5,-3$ and -1.4 for $\mathrm{T}_{\mathrm{e}}=10,20,50$ and $100 \mathrm{Kev}$ respectively. The radiation is strongly polarised ranging from being circular along the field to linear perpendicular to the field.

- For $\Lambda_{\mathrm{s}} \sim 10^{5}-10^{8}$ the lower harmonics are self absorbed. As a consequence the spectrum at low harmonics is of the black body Rayleigh-Jeans type $\left(\mathrm{I}_{\omega} \propto \omega^{2}\right)$ and is unpolarised (Figure 3 ).

- The radiation is strongly angle dependent being beamed perpendicular to the magnetic field. The beaming effect is strongest at high harmonics.

The point source models were successful in explaining the general characteristics of the observations, such as the presence of linear pulses near $\theta \sim 90^{\circ}$ and the strong angular dependence of intensity and circular polarisation. They failed in three major respects. Firstly, they were unable to explain the flat $\left(I_{\omega} \propto \omega^{-1-0}\right)$ polarized energy distributions seen over an extended wavelength region in some systems, such as AM Herculis itself. Secondly, the values of $\Lambda$ deduced from the 
optical cyclotron spectra were typically a factor of $\sim 100$ smaller than the values that were required to explain the $\mathrm{X}$-ray luminosities. Finally, the models predicted symmetrical light and polarisation curves which were contrary to observations.

Recently cylindrically symmetric models have been constructed which allow for density and temperature variations across the shock in response to a decrease in the specific accretion rate away from the axis of the cylinder (Wickramasinghe and Ferrario (1988a), Stockman (1988), Wu and Chanmugam (1988)). In the central regions the shock in bremsstrahlung dominated and the electron temperature approaches the adiabatic shock value. If the accretion rate drops sufficiently rapidly away from the center, the shock becomes dominated by cyclotron cooling in the outer regions and the electron temperature falls below the adiabatic shock value. Wickramasinghe and Ferrario (1988a) have modelled the density variations by assuming a central core ( $\mathrm{r} \leq$ $\left.r_{0}\right)$ with constant $\Lambda_{h}$ and an outer region $\left(r>r_{0}\right)$ with a " $\Lambda$ profile" specified by $\Lambda_{h}(r) \propto r^{-\delta}$, where $r$ is the distance away from the axis of the cylinder. Here, $h$ is the shock height perpendicular to the surface and $\delta$ is an index determined by the accretion profile and the physics of the shock. The temperature variations have been modelled by assuming a constant $T_{e}\left(=T_{e}(0)\right)$ for $r \leq r_{0}$ and a linear drop in temperature from $T_{e}(0)$ to $T_{e}(1)$ for $r>r_{0}$. For a bremsstrahlung dominated shock $\mathrm{h} \propto \mathrm{N}_{\mathrm{e}}^{-1}$ and $\delta=0$ irrespective of accretion profile. Large values of $\delta$ are only possible in a cyclotron dominated shock, and then only if the accretion rate drops rapidly across the shock. The models (Figure 4) show that flat polarized energy distributions are predicted over an extended frequency range, for large values of $\delta(\sim 4-6)$.

Wickramasingle and Ferrario (1988a) also showed that by offsetting the cylindrical emission region from the magnetic pole, asymmetric light and polarisation curves can be produced. However, the asymmetries were not large enough to explain observations of systems such as VV Puppis and V834 Cen .

From our discussion in $\$ 2.1$ it is evident that the emission region is more likely to have linear structure than cylindrical structure. Wickramasinghe and Ferrario (1986) have recently carried out calculations of ribbon like emission regions on the surface of a white dwarf with a dipolar field structure. The emission region is approximated by a strip in the magnetic colatitude $(\theta)$ - magnetic longitude $(\psi)$ plane defined by $\theta_{1}, \theta_{2}, \theta_{2}{ }^{*}$ and $\psi_{1}, \psi_{2} . \psi=0, \pm \pi$ on the plane through the dipole axis perpendicular to the orbital plane. The strip has an angular thickness $\Delta \theta=\left(\theta_{1}-\theta_{2}\right)$ and follows lines of constant magnetic colatitude if $\theta_{2}=\theta_{2}{ }^{*}$. Models have been constructed for a range of parameters assuming a constant value of $T_{e}(=10 \mathrm{Kev})$ and $\Lambda_{h}\left(=10^{5}\right)$ across the shock. The results (Figure 5) show that ribbons produce larger asymmetries in the polarisation and intensity curves than offset cylindrical emission regions. We note the following 
- the projected area effect results in a double humped light curve even for regions that are not extended. However, strongly asymmetric double humped light curves are calculated only for strips with a significant angular extent in $\psi$.

- for extended strips, the pulses need not coincide with the intensity maxima.

- for strips that are significantly elongated in magnetic latitude, partial eclipses produce triangular type intensity profiles.

The above characteristics have been noted in V834 Cen by Cropper (1988) who discussed the possibility of an accretion ribbon. However it should not be concluded that significant linear extension is a common property of the shocks in all AM Her type systems. The amount of extension is likely to depend critically on the dipole inclination $(\S 2)$.

\section{\$2.4 Two pole emission}

Several AM Her variables show a reversal in the sign of circular polarisation for part of the rotational phase (Bailey (1987)). The circular polarisation will change sign if the projection of the field along the line of sight changes sign. The reversals could be due to (a) a significant shock height above the stellar surface (b) an inclined (non radial) field direction at the location of the shock or (c) the presence of a second cyclotron emission region on the white dwarf surface of opposite field polarity. The recent detection of 3 linear pulses in EF Eri, AM Herculis and VV Puppis (Piirolla (1988) has shown beyond any doubt that there are some systems in which a second emission region makes an important contribution to the polarized radiation. It seems likely that the observed reversals in most systems are mainly due to (b) and (c), the shock height $\left(\mathrm{h} / \mathrm{R}_{\mathrm{wd}} \lesssim 0.1\right)$ being generally small in comparison to its extension across the surface (Wickramasinghe (1988), Stockman (1988)).

We illustrate the characteristics of two pole emission by presenting in Figure 6 the predictions of the model for AM Herculis based on linear polarisation angle data and the assumption that accretion occurs onto the foot points of closed field lines ( $\$ 2.2)$. The contributions from the two poles are presented separately without eclipses in the top right panel, and with eclipses in the bottom left panel. The pole which is eclipsed between $\phi=0.0-0.2$ is the main (hard X-ray) pole and is responsible for circular polarisation of negative sign during the bright phase. The second pole (the soft X-ray pole) is visible between phases $\phi \approx 0.0-0.4$ and contributes circular polarisation of opposite sign. The second zero crossing of circular polarisation is determined by a balance between the two poles and is expected to vary with epoch, precisely as observed. An intriguing feature of the model is that both poles contribute a linear pulse at phase $\phi=0.0$. In fact, it is likely that the pulse at $\phi=0.0$, which has traditionally been attributed to the main pole, may in fact originate mainly from the second pole. 


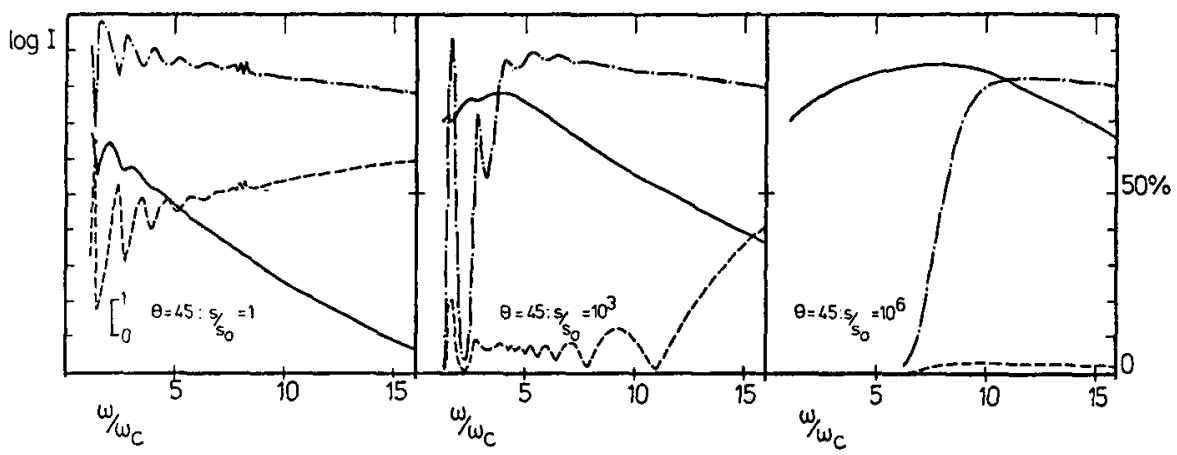

Figure 3 Radiative transfer effects on the intensity and polarisation spectra for $\mathrm{T}_{\mathrm{e}}=20 \mathrm{Kev}$ for various $\Lambda=\mathrm{s} / \mathrm{s}_{0}$. The dashed curves and dash-dotted curves are percentage linear and circular polarisation respectively.
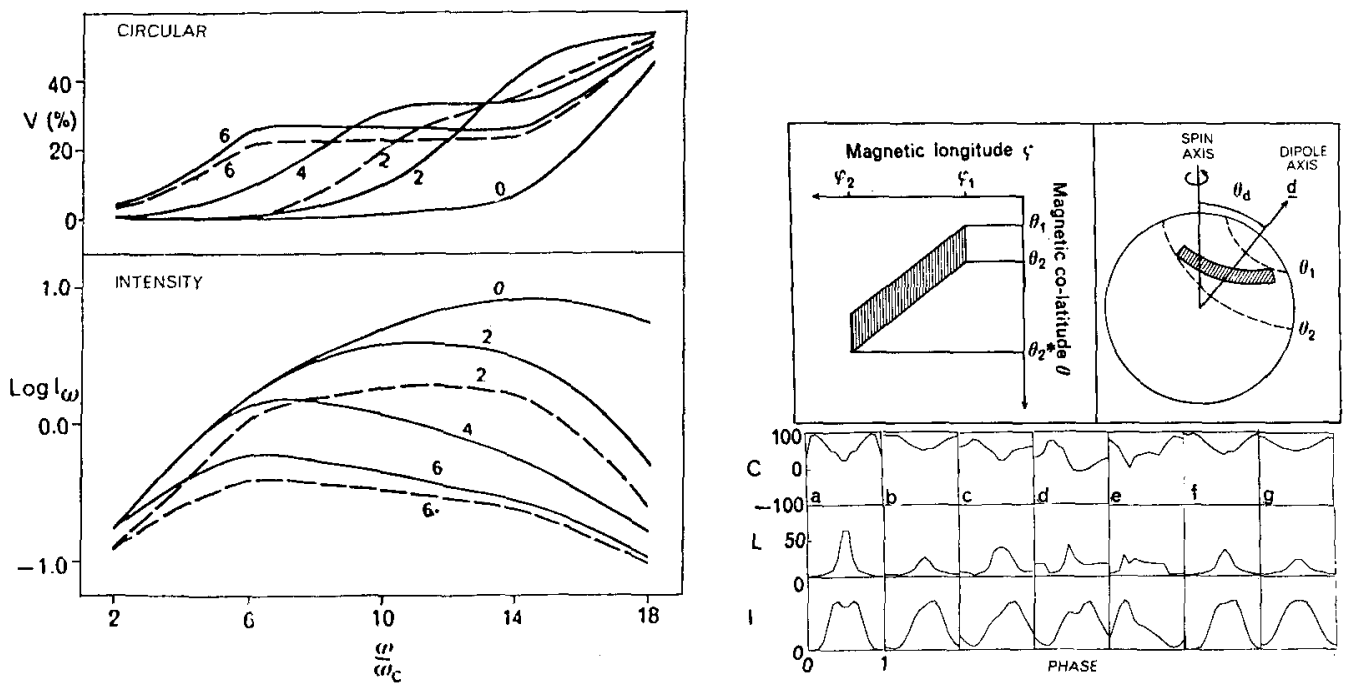

Figure 4 Left panel :Intensity and circular polarisation for cylindrically symmetric shocks. The curves are labelled by $\delta$. The models have $\mathrm{T}_{\mathrm{e}}(0)=10 \mathrm{Kev}$, $\Lambda_{h}(0)=210^{8}$ and are viewed at $i=60^{\circ}$ to the axis of symmetry. The dashed curve correspond to models with a declining temperature with $\mathrm{T}_{\mathrm{e}}(1)=10 \mathrm{Kev}$.

Figure 5 Right panel : Intensity (I) linear (L) and circular (C) polarisation for ring type geometries. The models have $\mathrm{T}_{\mathrm{e}}=10 \mathrm{Kev}, \Lambda_{\mathrm{h}}=10^{5}, i_{\text {orb }}=50^{\circ}$, $\delta_{d}=20^{\circ}$ and $\omega / \omega_{c}=9$. The models are labelled by $\theta_{1}, \theta_{2}, \theta_{2}{ }^{*}, \psi_{1}, \psi_{2}$

(a) $10,15,15,-2,+2$ (b) $10,15,15,0,180$ (c) $10,15,35,0,180$ (d) $10,15,55,0,180$

(e) $10,15,45,-180,0$ (f) $10,15,15,0,90$ (g) $10,15,15,0,360$. 


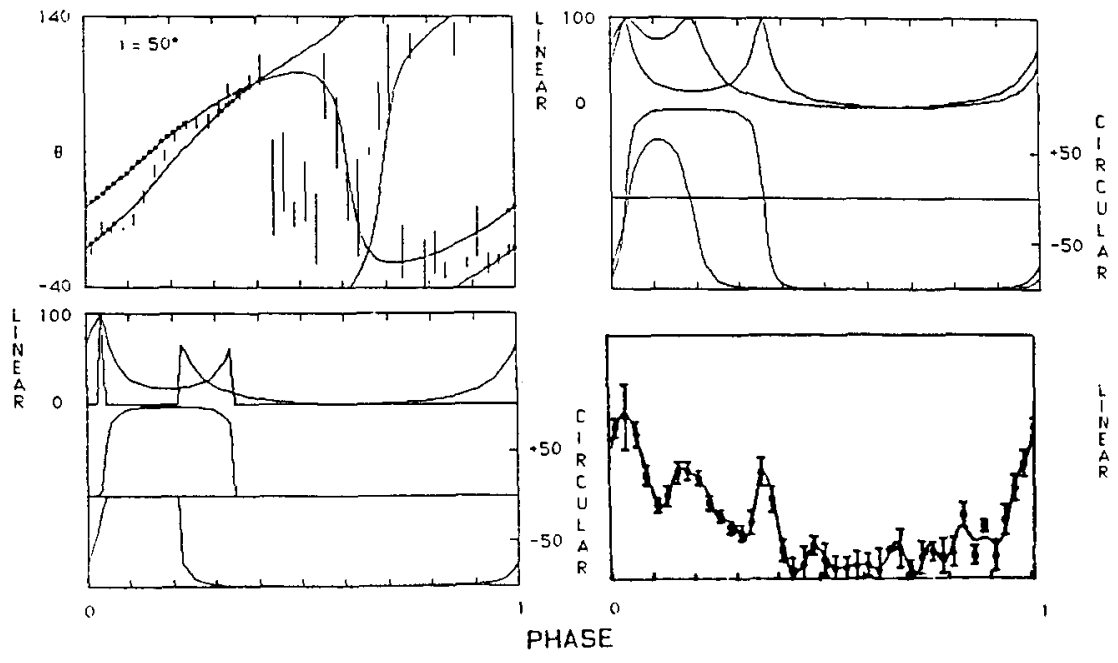

Figure 6 Fits to the linear polarisation angle data on AM Herculis from Piirola (1988) illustrating the two spot model. The spot positions are deduced on the assumption of accretion along closed field lines in a dipole field distribution.

\section{§3. Conclusions}

We have presented a brief review of some of the new developments that have occurred in the theory of the AM Her variables during the past two years. The main results are as follows :

- The specific accretion rate and hence the temperature and density are likely to vary across the shock. It is possible to construct structured shock models in which the hard X-rays originate from a compact high density core (with $\Lambda_{\mathrm{h}} \sim 10^{8}$ ) and the optical and near IR radiation from a surrounding lower density region (with $\Lambda_{h} \sim 10^{5}-10^{7}$ ). These models yield energy distributions that are in closer agreement with observations than previous point source models.

- The shocks may exhibit linear ribbon like extension across the surface. Thus geometrical effects could be important in interpreting polarisation and intensity light curves.

- Most systems have two active cyclotron emission regions displaced by $\sim 10^{\circ}$ from the magnetic poles. Both regions are simulateneously visible for part of the rotational cycle.

We have shown that by combining these effects, many of the previously unexplained phenomena can be interpreted, at least in a semi quantitative manner. 


\section{References}

Bailey, J., 1988 in "Polarized radiation of circumstellar origin", G. Coyne, A. Magalhaes, A.F.J. Moffat, R.E. Schulte-Ladbeck, S. Tapia and D.T. Wickramasinghe, eds; Vatican press.

Cropper, M., 1988, Mon. Not. Roy. Ast. Soc. (in press).

Ferrario, L., and Wickramasinghe, D.T., 1988 (these proceedings).

Ferrario, L., Wickramasinghe, D.T. and Tuohy, I.R., 1988, Ap.J. (submitted)

Meggitt, S.M.A. and Wickramasinghe, D.T., 1988, Mon. Not. Roy. Ast. Soc. (in press).

Piirola, V., 1988 in "Polarized radiation of circumstellar origin" G. Coyne, et al. eds; Vatican press.

Schmidt, G., 1987, I.A.U. Coll. No.95 on Faint Blue Stars, A.G. Davis Philip, D.S. Hayes and J. W. Liebert, eds, L. Davis Press.

Schmidt, G., 1988 in "Polarized radiation of circumstellar origin" G. Coyne et al. eds, Vatican press.

Stockman, H.S., 1988 in "Polarized radiation of circumstellar origin" G. Coyne et al. eds, Vatican press.

Wickramasinghe, D.T., 1988 in "Polarized radiation of circumstellar origin" G. Coyne et al. eds, Vatican press.

Wickramasinghe, D.T., 1987, I.A.U. Coll. No 95 on Faint blue stars, A.G. Davis Philip, D.S. Hayes and J.W. Liebert, eds, L. Davis Press.

Wickramasinghe, D.T. and Martin, B., 1985, Mon. Not. Roy. Ast. Soc. 212, 353.

Wickramasinghe, D.T. and Ferrario, L., 1988a, Ap. J. (in press)

Wickramasinghe, D.T. and Ferrario, L., 1988b, Ap. J. (in preparation)

Wickramasinghe, D.T., Visvanathan, N.V. and Tuohy, I.R., 1987, Ap. J. 318, 326.

Wu, K. and Chanmugam, G., 1988, Ap. J. (in press.) 